\title{
Grid sequence detector based on a stationary reference frame
}

\author{
Eider Robles, Josep Pou*, Salvador Ceballos, Igor Gabiola, and Maider Santos \\ ROBOTIKER-TECNALIA \\ Parque Tecnológico Edif. 202 \\ 48170 Zamudio (Bizkaia), Spain \\ Phone: +34 (94) 600-2266; Fax: +34 (94) 600-2299 \\ Email: erobles@ robotiker.es \\ URL: http://www.robotiker.com \\ * TECHNICAL UNIVERSITY OF CATALONIA \\ Department of Electronic Engineering \\ C/ Colom, no. 1 \\ 08222-Terrassa, Catalonia, Spain \\ Tel.: +34 / 937398306; Fax: +34 / 937398016 \\ E-Mail:pou@eel.upc.edu \\ URL: http://www.upc.edu
}

\section{Acknowledgments}

This work was supported by the Ministerio de Ciencia e Innovación of Spain under the Project CEBATE (ENE 2007-67033-C03-01/ALT).

\section{Keywords}

$<<$ Sequence detector $>>$, $<<$ moving average filter $>>,<<$ PLL $>>,<<$ grid connected power converters $>>$, $<<$ grid voltage synchronization $>>$.

\begin{abstract}
This paper proposes a new three-phase positive sequence detector. The scheme is based on a stationary reference frame and a Moving Average Filter (MAF) that guarantees the complete cancellation of harmonics and grid imbalances. The performance of the MAF is mathematically analyzed and a proper selection of the optimal filter's window width is realized. The proposed detector operates in open loop and there is no PI controller to be tuned. Thus, the dynamic response and simplicity is improved compared to other solutions. Performance of the proposed detector is verified through simulation and experiment. It shows very good performance under extreme grid voltage conditions, allowing fast detection of the grid voltage positive sequence (within one grid voltage cycle).
\end{abstract}

\section{Introduction}

Grid voltage synchronization is nowadays one of the most important issues in the connection of power converters to grid. Detection of the fundamental-frequency positive-sequence component of the utility voltage under unbalanced and distorted voltages is needed for a proper operation of grid-connected power electronic systems. Many synchronizing methods have been presented during the last years. The simplest ones are based on the detection of the peak value and zero crossing instants. However these methods might lead to significant angle and magnitude errors when the grid voltages are unbalanced and/or distorted.

Some extended methods are based on a phase-locked loop (PLL) including a feedback loop with the purpose of controlling a magnitude which is dependent on the voltage phase angle. They are applied to both, single-phase and three phase systems. Some single phase PLL examples can be found in [1]-[5]. 
PLLs are possibly the most extended synchronization methods in the case of grid-connected three-phase systems and are usually based on the use of a synchronous reference frame [6], [7]. The dynamic response of some methods, such as the one in [8], is good under balanced grid voltages, but very slow when they are designed to operate under grid voltages that are unbalanced or distorted. The algorithm presented in [9] copes this drawback and can cancel out the influence of certain harmonics; however, its implementation is highly complex and should be limited to cancel the effect of only a few harmonics. A PLL-based synchronization method is used in [10] which shows good performance, even under voltage imbalances and distortion. However, its implementation requires solving some practical problems related to the normalization of the detected angle.

There are other kind of synchronization algorithms based on the instantaneous symmetrical components theory [11]-[15]. In particular, [11] presents a simple and fast execution algorithm that allows the voltage positive sequence detection and system synchronization. It presents good performance when the grid has no harmonics, otherwise the response is significantly deteriorated. The method in [12] uses notch filters to improve its performance in the presence of harmonics. The method in [13] can perform well in the presence of grid frequency changes and imbalances. It is based on a specific filter called second order generalized integrator (SOGI). Its main drawback is that voltage grid harmonics are only attenuated but not completely canceled. An alternative method [15] also applies the symmetric components theory and extracts the fundamental frequency positive sequence by performing simple calculations. It completely eliminates several harmonics and employs finite impulse response filters to attenuate the rest.

The use of moving average filters in the detection of the positive sequence is relatively recent [16]-[19]. They are perfect filters when the window width is properly selected, but their use inside the closed loop of a PLL may slow down the response. The synchronization method presented in this paper is based on a stationary reference frame and moving average filters. The variables are properly processed to obtain the phase of the grid voltage positive sequence. Park transformations are used to obtain the positive and negative sequence components. This method allows fast detection of grid sequences and is no longer affected by the presence of imbalances or harmonics in the electrical grid.

\section{Grid Voltage Characterization}

Grid voltages can be considered a positive sequence with the addition of a negative and zero sequence (unbalanced voltages). They can also include harmonic components which can be either balanced or unbalanced (harmonic distortion). The objective of a positive sequence detector is to determine the magnitude and phase of the positive sequence fundamental component disregarding all unwanted components in the grid voltages.

A simplified representation of the grid voltages containing only the fundamentals and a harmonic set, which can be a positive or negative sequence, is given in the following:

$$
\left[\begin{array}{c}
v_{a} \\
v_{b} \\
v_{c}
\end{array}\right]=\widehat{V}_{+1}\left[\begin{array}{c}
\cos (\omega t) \\
\cos (\omega t-2 \pi / 3) \\
\cos (\omega t+2 \pi / 3)
\end{array}\right]+\widehat{V}_{n}\left[\begin{array}{c}
\cos \left(n \omega t+\theta_{n}\right) \\
\cos \left(n \omega t-2 \pi / 3+\theta_{n}\right) \\
\cos \left(n \omega t+2 \pi / 3+\theta_{n}\right)
\end{array}\right]
$$

in which $n$ is an integer number from $-\infty$ to $+\infty$ different from $+1 . \widehat{V}_{+1}$ and $\widehat{V}_{n}$ are the amplitudes of the fundamentals and the harmonics, respectively. Note that for $n=-1$, the second term in (1) corresponds to a fundamental negative sequence.

The voltage vector can be represented in $\alpha \beta$ coordinates through the Clarke transformation, using a stationary reference frame. If this transformation is applied to the voltages in (1), the following $v_{\alpha}$ and $v_{\beta}$ components are obtained:

$$
\left[\begin{array}{c}
v_{\alpha} \\
v_{\beta}
\end{array}\right]=\widehat{V}_{+1} \sqrt{\frac{3}{2}}\left[\begin{array}{c}
\cos (\omega t) \\
\sin (\omega t)
\end{array}\right]+\widehat{V}_{n} \sqrt{\frac{3}{2}}\left[\begin{array}{c}
\cos \left(n \omega t+\theta_{n}\right) \\
\sin \left(n \omega t+\theta_{n}\right)
\end{array}\right]
$$

Under ideal grid voltages, $v_{\alpha}$ and $v_{\beta}$ are two sinusoidal waveforms dephased $\pi / 2$ whose amplitude is $\widehat{V}_{+1} \sqrt{\frac{3}{2}}$.

Fig. 1.a shows grid voltages distorted by a dephased negative sequence $(10 \%)$ and a $7^{\text {th }}$ order harmonic $(20 \%)$. Fig. 1.b shows the evolution of the voltage vector on the $\alpha \beta$ diagram. The fundamental frequency positive sequence voltage vector adds up with a fundamental frequency negative sequence vector and a vector caused by the $7^{\text {th }}$ order harmonic. Being $\mathbf{V}$ the grid voltage vector, $v_{\alpha}$ and $v_{\beta}$ the projections of $\mathbf{V}$ on the $\alpha \beta$ plane and $\theta^{\prime}$ the position angle of $\mathbf{V}$. This angle can be obtained by performing a simple $\operatorname{arctangent}\left(v_{\beta} / v_{\alpha}\right)$ operation to the transformed input voltage variables. When the grid voltages are ideal (balanced and undistorted), $\theta^{\prime}$ will correspond to the positive-sequence angle $\theta$. 

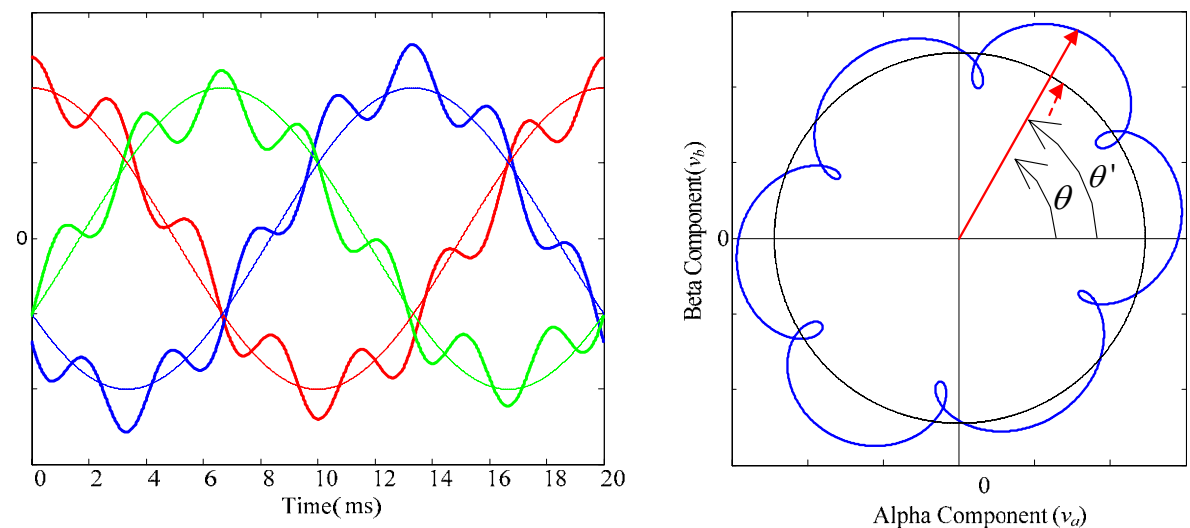

Figure 1: Voltage vector on the $\alpha \beta$ plane.

On the other hand, according to (2), voltage imbalances and distortion will cause oscillations in the two components $\left(v_{\alpha}\right.$ and $\left.v_{\beta}\right)$ and therefore, in the arctangent calculation. In this case, $\theta^{\prime}$ will not represent the positive sequence phase angle if the oscillations are not previously removed. An $n^{\text {th }}$ order harmonic in the $a b c$ frame and in the transformed $v_{\alpha}$ and $v_{\beta}$ variables, will cause infinite harmonic components in the performed arctangent, and therefore in the phase angle. The main frequency order of these oscillations will be $(n-1)$. Hence, the main influence of imbalances and distortion in the detected angle is known and corresponds to the following:

- Odd-order harmonics generate even-order frequency oscillations in in the performed arctangent and therefore, phase angle will only depend on even-order sinusoidal components.

- Even-order harmonics generate both even and odd-order frequency oscillations in the performed arctangent and therefore, phase angle will depend on both order sinusoidal components.

The negative sequence caused by voltage dips also generates even-order sinusoidal components on the phase angle, and it can be considered as a negative sequence first order harmonic.

To obtain the real angle of a positive sequence, the oscillating components have to be removed. A MAF structure may be very useful because, under certain conditions, it can perform as an ideal low-pass filter. Performance of the MAF is analyzed in the following.

\section{Moving Average Filter (MAF)}

Application of the MAF operator to an input signal $x(\tau)$ is given by

$$
\bar{x}(t)=\frac{1}{T_{w}} \int_{t-T_{w}}^{t} x(\tau) d \tau .
$$

A performance example of the MAF is shown in Fig. 2. The filter provides the mean value of the input signal in the time period from $t-T_{w}$ to $t$, where $T_{w}$ is the window width. There is always an intrinsic delay related to the window width; therefore, the larger $T_{w}$, the slower the detection dynamic. If the input signal contains sinusoidal components of an integer multiple of the equivalent frequency of the MAF $\left(f_{w}=1 / T_{w}\right)$, these components can be completely removed and therefore, the output signal will be a constant value. The MAF will need $T_{w}$ time to gather the data and obtain the correct output, hence this is the maximum delay in correcting the output in the presence of input transitories.

In Fig. 2 the window width is $0.01 \mathrm{~s}$, exactly the same as the input signal period. In this case, it could correspond to a negative sequence produced by a voltage dip. The MAF can eliminate the input oscillation after gathering the data. When the input signal changes, it has a slight error during $T_{w}$ until it has again an integer number of oscillations and can perform the mean value.

Selection of the window width is an important issue. Depending on it, the MAF is able to perform as an ideal low-pass filter for some input frequency components.

For a better understanding of the MAF the transfer function has been obtained and analyzed. Given an input signal:

$$
x(t)=A \sin \left(\omega t+\theta_{0}\right),
$$




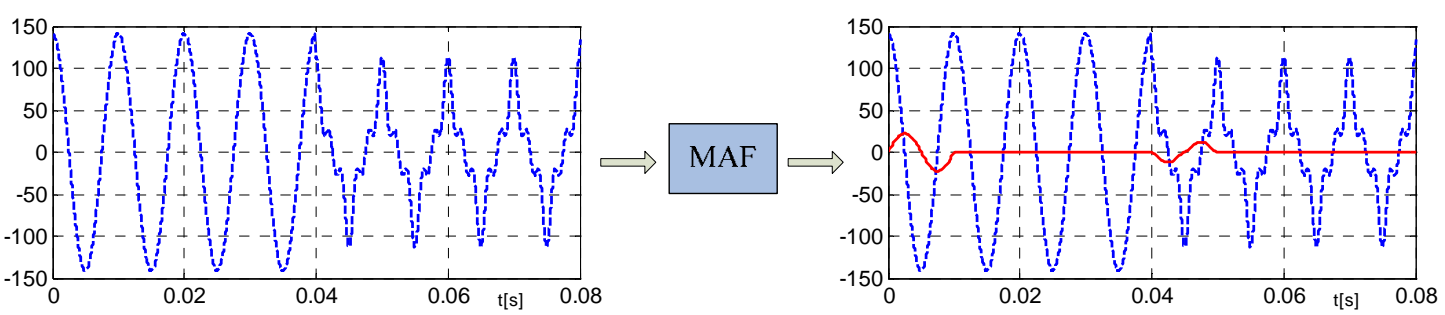

Figure 2: Performance example of the MAF.

with $\omega=2 \pi / T$, the output of the MAF will be:

$$
\bar{x}(t)=\frac{A}{\omega T_{w}}\left\{\cos \left[\omega\left(t-T_{w}\right)+\theta_{0}\right]-\cos \left(\omega t+\theta_{0}\right)\right\} .
$$

Applying the Laplace transform to (4) and (5) the transfer function for $\theta_{0}=0$ becomes as follows:

$$
G_{M A F}(s)=\frac{\bar{X}(s)}{X(s)}=\frac{s\left[\cos \left(\omega T_{w}\right)-1\right]+\omega \sin \left(\omega T_{w}\right)}{\omega^{2} T_{w}} .
$$

Substituting $s=j \omega$ :

$$
G_{M A F}(j \omega)=\frac{\bar{X}(j \omega)}{X(j \omega)}=\frac{j\left[\cos \left(\omega T_{w}\right)-1\right]+\sin \left(\omega T_{w}\right)}{\omega T_{w}} .
$$

The magnitude and phase expressions are respectively:

$$
\begin{aligned}
& \left|G_{M A F}(j \omega)\right|=\frac{\sqrt{2}}{\omega T_{w}} \sqrt{1-\cos \left(\omega T_{w}\right)} \text { and } \\
& \varphi\left[G_{M A F}(j \omega)\right]=\arctan \left[\frac{\cos \left(\omega T_{w}\right)-1}{\sin \left(\omega T_{w}\right)}\right] .
\end{aligned}
$$

From (8), the output magnitude of the MAF is zero whenever $\left[1-\cos \left(\omega T_{w}\right)\right]=0$, that is:

$$
\omega T_{w}=k 2 \pi \text { for } k=1,2,3, \ldots
$$

or:

$$
\frac{T_{w}}{T}=\frac{f}{f_{w}}=k \text { for } k=1,2,3, \ldots
$$

Fig. 3 shows the Bode diagram of this transfer function for a window width of $T_{w}=0.02 \mathrm{~s}$. Observe that the frequency components meeting (11) are completely canceled. In this particular case, it will remove all the harmonics of a $50 \mathrm{~Hz}$ input signal.

If the average value of the input signal remains constant, and $T_{w}$ is properly adjusted, the output of the MAF does not produce any steady-state error. However, if the input signal increases or decreases with a constant slope $x(t)=m \cdot t$, the output of the MAF includes a constant error given by $d=\frac{m T_{w}}{2}$ (Fig. 4). Consequently, this value has to be added up to the output of the MAF to compensate for such an error.

\section{Stationary Frame Sequence Detector (SFSD)}

Fig. 5 shows the structure of the proposed positive sequence detector. By means of the Clarke transformation, the input voltage vector is transformed into $\alpha \beta$ components. These transformed components will be sinusoidal and may contain oscillations due to harmonics and a negative voltage sequence. A four-quadrant arctangent operation is performed and the input voltage phase angle is detected. As well as the $\alpha \beta$ components, this phase angle may contain oscillations. It is thus filtrated through a MAF. 


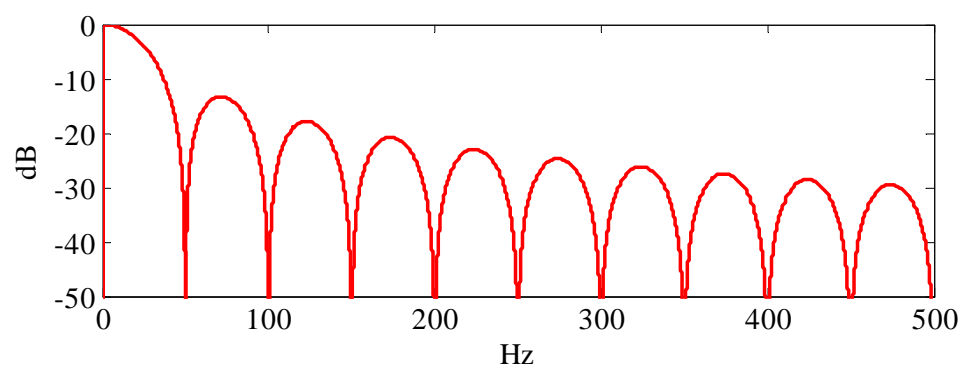

Figure 3: Bode diagram of the MAF.

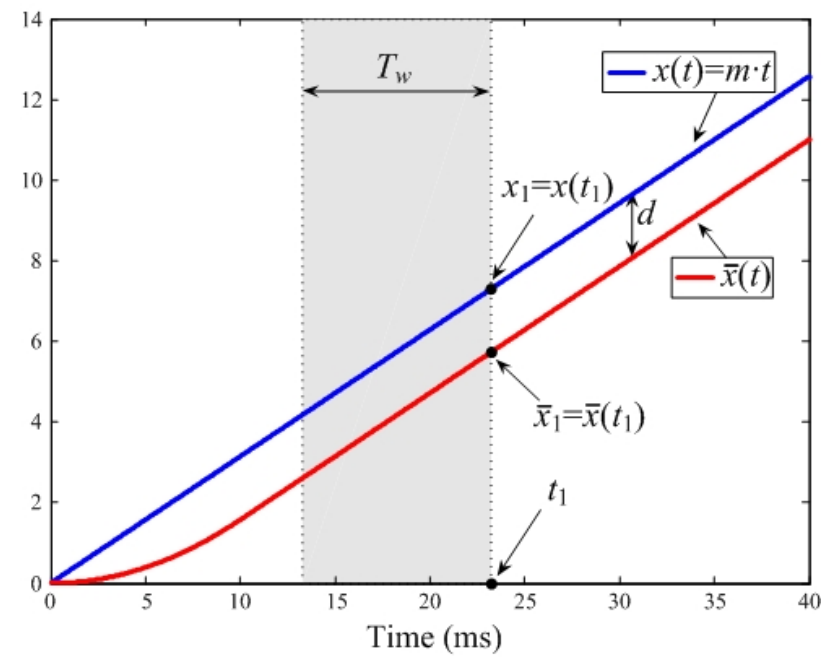

Figure 4: Performance of the MAF with slope inputs. 


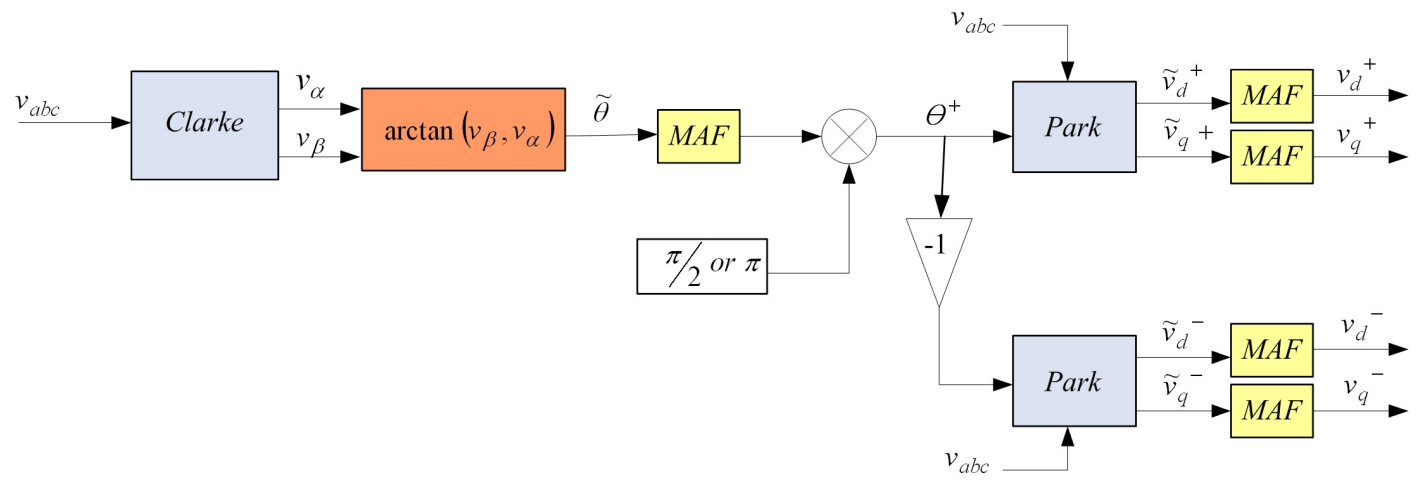

Figure 5: Structure of the proposed PLL.

Since the angle increases continuously with a constant slope, the error produced by the MAF has to be compensated.

If the window width is $T_{w}=T_{g}$, being $T_{g}$ the grid voltage period, the correction constant to compensate for the angle error is $\pi$. Similarly, the compensation angle is $\pi / 2$ if the selected window width of the MAF is $T_{w}=T_{g} / 2$. Once the error is compensated, the positive sequence angle $\theta^{+}$is obtained free from oscillations due to disturbances and imbalances.

Once the positive angle is detected, it is possible to obtain both, the positive and the negative sequences by applying the Park transformation to the original $a b c$ input voltages with $\theta^{+}$for the positive sequence and $\theta^{-}=-\theta^{+}$for the negative sequence. The obtained $d q$ components may contain oscillations that can be removed using again a MAF. In this case, it is not necessary to compensate for steady-state errors because the average voltage of the variables remain constant in the steady state.

An interesting feature of this detection method is its simplicity. Furthermore, note that there are not PI regulator parameters or any other coefficients that need to be tuned. This is a significant advantage compared to other positive-sequence detectors. Additionally, since there are no feedback loops in this scheme, the detector is always stable.

\section{Simulation Results}

Some simulation results from the proposed SFSD are shown in this section. In all the simulations the rms line-to-neutral grid voltage is $220 \mathrm{~V}$ at $50 \mathrm{~Hz}$.

Fig. 6, shows the system response in the presence of odd-order harmonics. The harmonic components are added at $t=30 \mathrm{~ms}$ as follows: $5^{t h}$ order $(50 \%), 11^{\text {th }}$ order $(30 \%)$ and $19^{\text {th }}$ order $(20 \%)$. For a better understanding of the proposed detector, this figure shows internal variables of the detector scheme, as follows: (a) Grid voltages, (b) $\alpha \beta$ components, (c) performed arctangent, (d) detected angle (blue) vs. real angle (red), (e) error in the detected angle, (f) voltage vector projection on $d q$ positive axes, (g) positive sequence $d q$ components filtrated through MAF, (h) voltage vector projection on $d q$ negative axes, (i) negative sequence $d q$ components filtrated through MAF, and (j) grid voltages positive sequence. As only odd-order harmonics are expected, a window width of $T_{w}=T_{g} / 2$ is used. Results show a fast and good performance of the SFSD. There is only a slight error when the harmonic components appear and disappear while the MAF is gathering the data (half signal period, $T_{w}$ ). The positive and negative sequence components are also properly filtrated.

Fig. 7 shows the performance of the SFSD in the presence of voltage dips. At $t=30 \mathrm{~ms}$ two phases decrease up to $20 \%$ and the third up to zero. Moreover, a $\pi / 5$ phase jump is added at $t=30 \mathrm{~ms}$. The voltage dip lasts until $t=70 \mathrm{~ms}$. Again a window width of $T_{w}=T_{g} / 2$ is used in the MAFs, enough to filter the negative sequence of the voltage dip. In this case, (a) grid voltages, (b) detected angle (blue) vs. real angle (red), (c) error in the detected angle, and (d) and (e) positive and negative sequence $d q$ components filtrated through MAF are shown. The error is corrected in a $T_{w}$ time and it is very small except for the phase jump where the error corresponds exactly to this phase difference.

In Fig. 8, the proposed positive-sequence detector is tested under extremely bad grid voltage conditions. At $t=30 \mathrm{~ms}$ a $\pi / 2$ phase jump, a three-phase voltage dip (20\%, $40 \%$ and $60 \%$ ), and a harmonic component set $5^{\text {th }}$ order $(50 \%), 7^{\text {th }}$ order $(25 \%), 13^{\text {th }}$ order $(20 \%)$ and $25^{\text {th }}$ order $(10 \%)$ are added simultaneously. At $t=70 \mathrm{~ms}$ the harmonics dissapear, and at $t=110 \mathrm{~ms}$ the voltage dip is recovered. The detector shows a very good performance and all the transitory errors are eliminated in half a grid period $\left(T_{w}=10 \mathrm{~ms}.\right)$ 

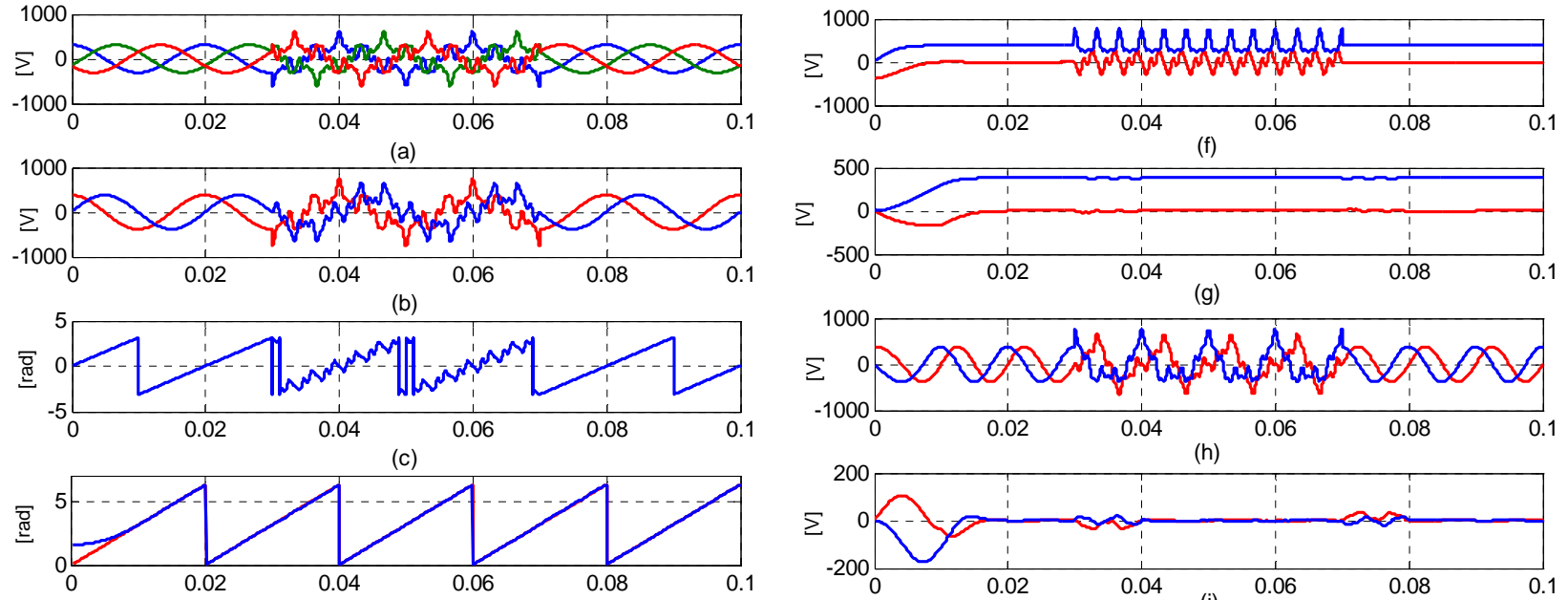

(d)
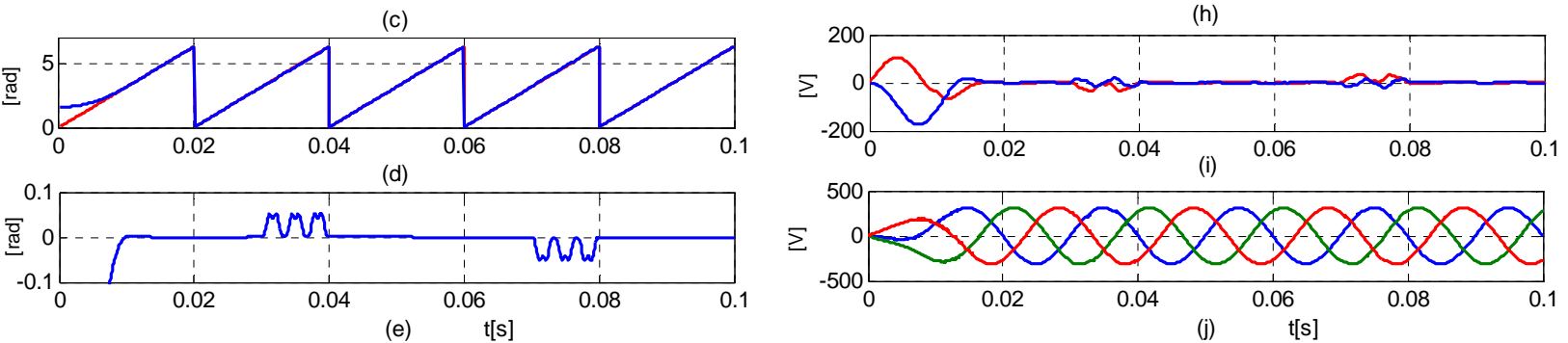

Figure 6: Simulation results. Odd order harmonic distortion.
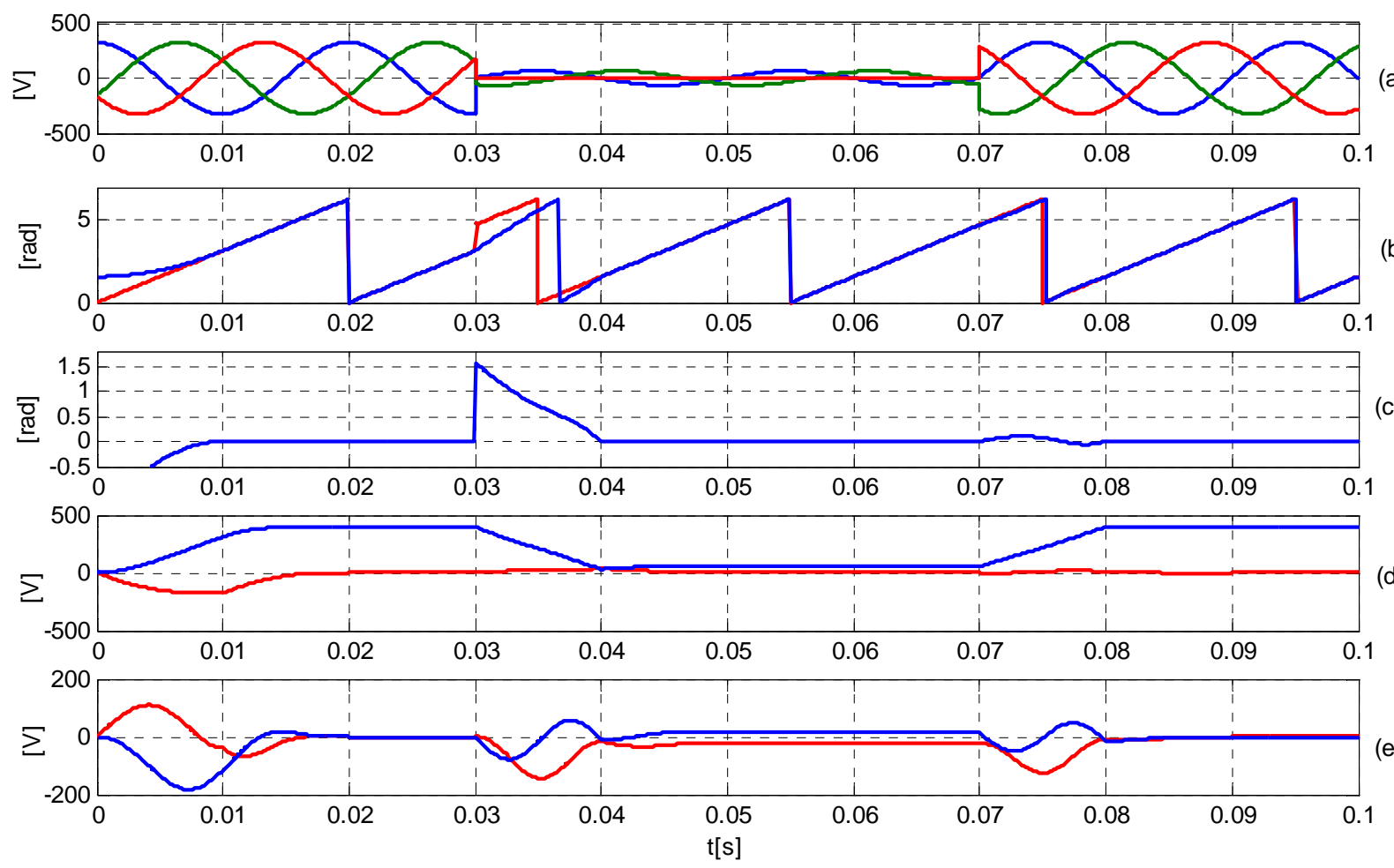

Figure 7: Simulation results. Three-phase voltage dip and phase jump. 

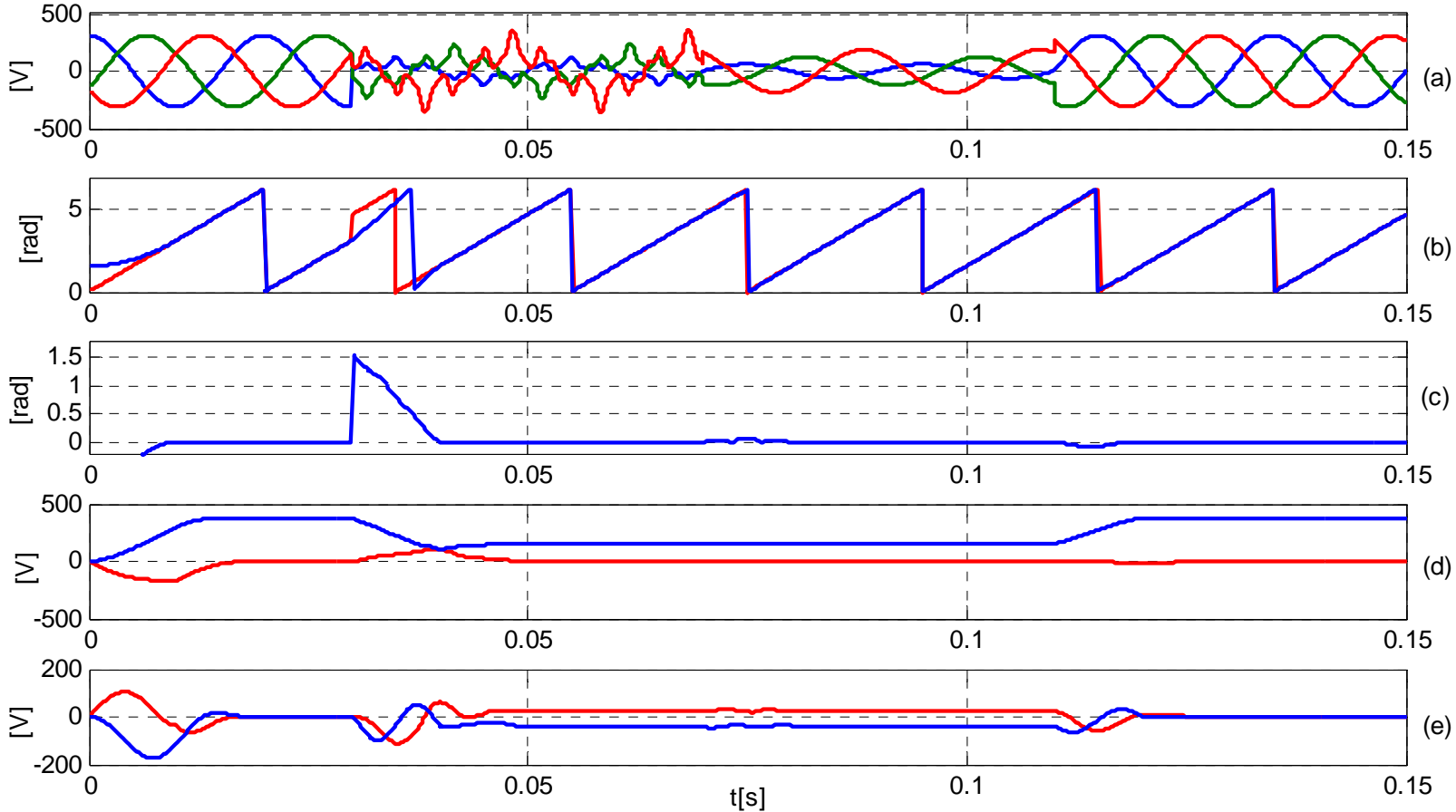

Figure 8: Simulation results. Behaviour of the proposed positive-sequence detector in the presence of voltage dips, phase jumps and harmonic distortion.

\section{Experimental Results}

The proposed algorithm was programmed in a DSP board TMS320F2812 and some experimental results were obtained. A three-phase generator CMC-156 from Omicron was used to provide the grid voltages. All the tests were performed with a line-to-neutral voltage of $50 \mathrm{Vrms} 50 \mathrm{~Hz}$. Fig. 9 presents the main variables of the proposed positive sequence detector. It shows (a) grid voltages, (b) detected phase angle, (c) positive-sequence $d q$ components, and (d) the obtained positive sequence of the grid voltages. The MAF window width is settled to $T_{w}=T_{g}(20 \mathrm{~ms})$ since even-order harmonics are expected.

At $t=60 \mathrm{~ms}$ the input voltage amplitude decreases to $65 \%$ and the first 25 harmonics are introduced as follows: Up to the $25^{\text {th }}$ harmonic according to $V_{h}=V_{\text {fundamental }} / h$ for $h=2,3 \ldots 25$. An imperceptible short error appears in the detected angle and it is corrected in a cycle time. The positive sequence $d$ component decreases due to the amplitude reduction. At $t=140 \mathrm{~ms}$ the harmonic set disappears, the amplitude of two of the phases recovers and the third phase becomes zero. The amplitude of the positive sequence becomes stable in a window width time, $T_{w}$. At $t=300 \mathrm{~ms}$, a phase jump of $\pi / 3$ is added in all the phases. Again, the error is corrected in $T_{w}$ time.

\section{Conclusion}

In this paper, a new three phase positive sequence detector has been presented. The structure is based on a stationary reference frame and a MAF that guarantees the complete cancelation of the effect of the harmonics and grid imbalances. Moreover there is no need for PI controllers, thus, the dynamic response of the system is improved compared to other solutions. Simulation and experimental results have been presented, which verify the good performance of the system in the presence of harmonics, imbalances and phase changes in the grid voltage. The positive sequence detector is designed for a determined frequency, but its performance is acceptable when slight frequency changes occur. Future work is focused on an adaptive structure able to operate under large frequency changes.

\section{References}

[1] M. Silva, B. M. Lopes, B. J. C. Filho, R. P. Campana, and W. C. Bosventura, "Performance evaluation of PLL algorithms for single-phase grid-connected systems," in Proc. Industry Applications Conference Annual Meeting 2004 (IAS'04), Oct. 2004, vol. 4, pp. 2259-2263. 

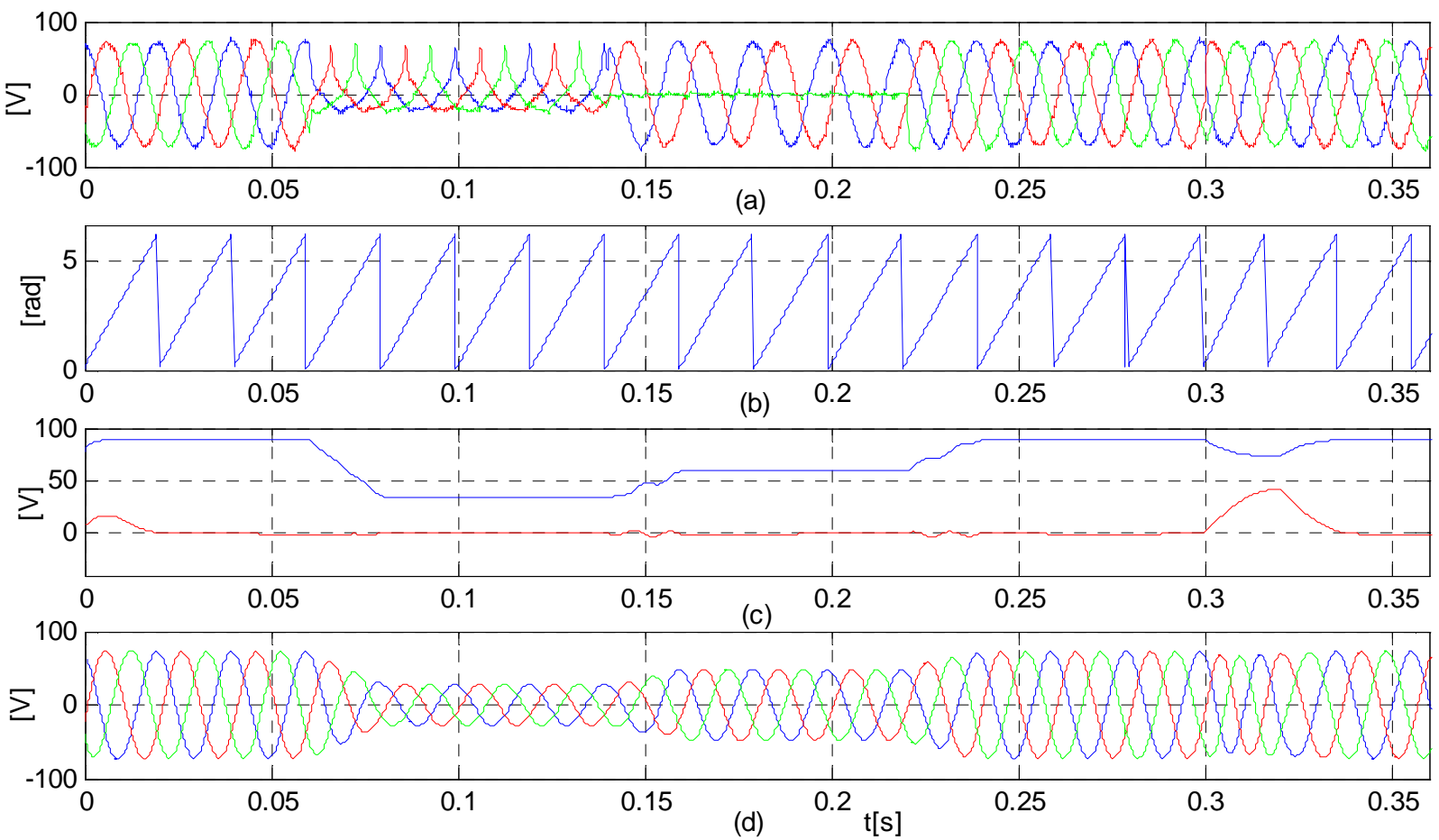

Figure 9: Experimental results. Voltage dip, even-order harmonics and phase jump.

[2] M. Karimi-Ghartemani and M. Reza Iravani, "A nonlinear adaptive filter for online signal analysis in power systems: applications," IEEE Trans. Power Delivery, vol. 17, no. 2, pp. 617-622, April 2002.

[3] M. Karimi-Ghartemani, H. Karimi, and M. Reza Iravani, "A magnitude/phase-locked loop system based on estimation of frequency and in-phase/quadrature-phase amplitudes," IEEE Trans. Ind. Electron., vol. 51, no. 2, pp. 511-517, April 2004.

[4] T. Thacker, R. Wang, D. Dong, R. Burgos, F. Wang, and D. Boroyevich, "Phase-Locked Loops using State Variable Feedback for Single-Phase Converter Systems," in Proc. APEC'09, Feb. 15-19, 2009, Washington, U.S.A, pp. 864-870.

[5] M. Ciobotaru, R. Teodorescu, and F. Blaabjerg, "A new single-phase PLL structure base on second order generalized integrator," in Proc. PESC'06, June 2006, vol. 4, pp. 1-6.

[6] D. Jovcic, ’Phase locked loop system for FACTS," IEEE Trans. Power Syst., vol. 18, no. 3, pp. 1116-1124, Aug. 2003.

[7] L. G. B. Barbosa, Rolim, D. R. Rodrigues, da Costa, Jr., and M. Aredes, "Analysis and software implementation of a robust synchronizing PLL circuit based on the pq theory," IEEE Trans. Ind. Electron., vol. 53, no. 6, pp. 1919-1926, Dec. 2006.

[8] S.-K. Chung, "A phase tracking system for three phase utility interface inverters," IEEE Trans. Power Electron., vol. 15, no. 3, pp. 431-438, May 2000.

[9] P. Rodríguez, J. Pou, J. Bergas, J. I. Candela, R. Burgos, and D. Boroyevich, ”Decoupled double synchronous reference frame PLL for power converters control,' IEEE Trans. Power Electron., vol. 22, no. 2, pp. 584-592, March 2007.

[10] J. Pou, E. Robles, S. Ceballos, J. Zaragoza, A. Arias, and P. Ibáñez, ”Control of Back-to-Back-Connected Neutral-Point-Clamped Converters in Wind Mill Applications," in Proc. EPE'07, Sept. 2-5, 2007, Dresden, Denmark.

[11] S. Alepuz, S. Busquets, J. Bordonau, J. Pontt, C. Silva, and J. Rodríguez, "Fast On-Line Symmetrical Components Separation Method for Synchronization and Control Purposes in Three Phase Distributed Power Generation Systems," in Proc. EPE'07, Sept. 2-5, 2007, Dresden, Denmark.

[12] M. Karimi-Ghartemani and M. Reza Iravani, "A Method for Synchronization of Power Electronic Converters in Polluted and Variable-Frequency Environments," IEEE Trans. Power Systems, vol. 19, no. 3, pp. 12631270, Aug. 2004.

[13] P. Rodríguez, A. Luna, M. Ciobotaru, R. Teodorescu, and F. Blaabjerg, "Advanced Grid Synchronization System for Power Converters under Unbalanced and Distorted Operating Conditions," in Proc. IEEE IECON'06, Nov. 7-10, 2006, Paris, France, pp. 5173-5178.

[14] M. Karimi-Ghartemani and H. Karimi,"Processing of Symmetrical Components in Time Domain," IEEE Trans. Power Systems, vol. 22, no. 2, pp. 572-579, May 2007. 
[15] F. Bradaschia, J. P. Arruda, H. E. P. Souza, G. M. S. Azevedo, F. A. S. News, and M. C. Cavalcanti, ”A Method for Extracting the Fundamental Frequency Positive-Sequence Voltage Vector Based on Simple Mathematical Transformations," in Proc. IEEE PESC'08, Jun. 15-19, 2008, Rodhes, Greece, pp. 1115-1121.

[16] A. M. Salamah, S. J. Finney, and B. W. Williams, "Three-phase phase-lock loop for distorted utilities," in Electric Power Applications, IET, vol. 1, no. 6, pp. 937-945, Nov. 2007.

[17] A. Ghoshal and V. John, "A Method to Improve PLL Performance Under Abnormal Grid Conditions," in Proc. NPEC'07, Dec. 17-19, 2007, Indian Institute of Science, Bangalore, India.

[18] E. Robles, S. Ceballos, J. Pou, J. Zaragoza, and I. Gabiola, "Grid Synchronization Method Based on a QuasiIdeal Low-Pass Filter Stage and a Phase-Locked Loop,' in Proc. IEEE PESC'08, Jun. 15-19, 2008, Rodhes, Greece, pp. 4056-4061.

[19] F.D. Freijedo, J. Doval-Gandoy, O. Lopez, and E. Acha, ”A Generic Open-Loop Algorithm for Three-Phase Grid Voltage/Current Synchronization With Particular Reference to Phase, Frequency, and Amplitude Estimation,” IEEE Trans. Power Systems, vol. 24, no. 1, pp. 94-107, Jan. 2009. 